\title{
Use of cardiac magnetic resonance imaging in the diagnosis of Takotsubo cardiomyopathy: another imaging feature added
}

\author{
Ron Blankstein
}

Received: 13 December 2009/Accepted: 15 December 2009/Published online: 29 December 2009

(C) Springer Science+Business Media, B.V. 2009

Takotsubo cardiomyopathy, also known as apical ballooning syndrome, is a relatively rare acute cardiac syndrome which has a pathophysiology which is not well understood. Patients with this disorder (which is named after the Japanese word for an octopus trap which has a shape that resembles the apical ballooning) often present after an emotionally or physically stressful event with chest pain, electrocardiographic changes, and mild elevation of cardiac markers which are suggestive of an acute coronary syndrome. However, upon angiography, no obstructive coronary artery disease is identified. In the acute setting, echocardiography or ventriculography typically show an aneurysmal or dyskinetic apex and mid ventricle with preserved or hyperdynamic function at the basal ventricular level.

Editorial comment on the article of Joshi et al. (doi: 10.1007/s10554-009-9515-5).

\section{R. Blankstein ( $\square)$}

Department of Medicine (Cardiovascular Division), Brigham and Women's Hospital, Harvard Medical School, 75 Francis Street, Room Shapiro 5096, Boston, MA 02115, USA

e-mail: rblankstein@partners.org

\section{R. Blankstein}

Department of Radiology, Brigham and Women's Hospital, Harvard Medical School, 75 Francis Street, Room Shapiro 5096, Boston, MA 02115, USA
Interestingly, these marked changes are usually completely reversible and the majority of patients experience complete recovery and have a good long term prognosis. Nevertheless, numerous associated complications ranging from heart failure and atrial or ventricular arrhythmias to lethal complications such as LV free wall rupture and ventricular septal defects have been reported. In addition, some patients with midventricular level involvement may experience dynamic obstruction in the LV outflow (i.e. similar to the pathophysiology of hypertrophic cardiomyopathy) which can be associated with systolic anterior motion of the anterior mitral valve leaflet and mitral regurgitation.

The pathophysiological mechanism underlying takotsubo cardiomyopathy remain mostly unknown. Proposed mechanisms for the observed apical ballooning have included vasospasm of the epicardial coronary arteries, microvascular dysfunction, catecholamine induced myocardial stunning and coronary emboli or thrombosis with subsequent vessel recanulization. Mechanisms such as spasm or thrombosis seem less likely as the often large degree of ventricular dysfunction is only associated with a mild elevation in cardiac enzymes. While myocardial stunning following repetitive ischemia could conceivably account for such a discrepant pattern, another observation which weakens this hypotheses is that the wall motion abnormality observed with takotsubo cardiomyopathy often extends beyond the territory normally supplied by a single coronary artery. 
On the other hand, there is data that supports the role of catecholamine mediated cardiotoxicity as the basis for takotsubo cardiomyopathy. Wittstein and colleagues showed that patients with takotsubo cardiomyopathy have a two to threefold increase in catecholamine levels compared to those presenting with similar degree of heart failure or myocardial infarction [1]. Interestingly, in an animal model of takotsubo cardiomyopathy, pretreatment with an an $\alpha$ - and $\beta$-adrenergic antagonist prevents this condition [2]. Moreover, histological changes in takotsubo cardiomyopathy (e.g. contraction band necrosis and neutrophil infiltration and fibrosis) resemble those seen in catecholamine cardiotoxicity and are distinctly different from patterns associated with ischemia [3]. Lastly, a notable recently suggested hypotheses is that the increased levels of epinephrine result in a switch in intracellular signal trafficking from $\mathrm{G}(\mathrm{s})$ protein to $\mathrm{G}(\mathrm{i})$ protein signaling via the beta(2)-adrenoceptor [4]. Although this switch protects against the proapoptotic effects of intense activation of beta(1)-adrenoceptors, it is also negatively inotropic. According to this hypotheses, this effect is greatest at the apical myocardium, in which the beta-adrenoceptor density is greatest [4].

While there are no established diagnostic criteria for the diagnosis of takotsubo cardiomyopathy, it has been suggested that the presence of all of the following are required for the diagnosis: (a) Transient hypokinesis, akinesis, or dyskinesis of the left ventricular apical and/or midventricular segments, (b) absence of obstructive coronary artery disease or angiographic evidence of acute plaque rupture, and (c) New electrocardiographic abnormalities (ST segment elevation and/or $\mathrm{T}$ wave inversions) or troponin elevations. Moreover, conditions such as pheochromocytoma or myocarditis should be excluded [5].

From a clinical perspective, the diagnosis of takotsubo cardiomyopathy relies on recognizing the anatomical abnormalities described above in patients (typically women and often following intense emotional stress) who often present with a suspected acute coronary syndrome. Patients often undergo urgent invasive angiography, especially if they present with ST elevations, which shows no evidence of obstructive disease but ventriculography will demonstrate the presence of apical ballooning and/or mid wall hypokineiss.
However, there are multiple scenarios which can complicate this seemingly straightforward diagnosis. For instance, some patients may have a thrombotic occlusion which is then followed by recanulization of the coronary vessel. Unless the site of plaque rupture is identified, invasive angiography may be unrevealing. Equally challenging are cases in which patients with significant coronary atherosclerosis present with possible takotsubo cardiomyopathy. Finally, in some cases, distinguishing between myocarditis and takotsubo cardiomyopathy may not be straight-forward (e.g. patients with both conditions can have elevated cardiac enzymes in the absence of obstructive coronary artery disease). This may especially be true for patients who present after their wall motion abnormalities have partially improved. It is noteworthy that arriving at the correct diagnosis for many of these conditions is important as the subsequent management can be entirely different. For instance, patients who are ultimately found to have an acute coronary syndrome (even in the absence of obstructive coronary artery disease) will greatly benefit from anti-platelet and lipid lowering therapies. On the other hand, those with uncomplicated takotsubo cardiomyopathy can be managed conservatively.

Cardiac magnetic resonance imaging (CMR) offers a robust non-invasive tool which can be used to improve the diagnosis and management of patients with suspected takotsubo cardiomyopathy. This advanced imaging modality may be particularly helpful for scenarios such as above when multiple etiologies are considered. In addition to demonstrating the typical wall motion abnormalities associated with this condition, CMR can be used to definitively exclude other etiologies which may be clinically suspected such as scar or myocarditis.

A comprehensive CMR exam for suspected takotsubo cardiomyopathy will include an evaluation of left and right ventricular volumes and function as well as a comprehensive assessment of regional wall motion abnormalities. First pass perfusion can be used to assess for resting myocardial perfusion defects, which in patients with takotsubo cardiomyopathy are often present in the apex. But perhaps the most powerful par of the CMR exam for this condition is the use of late enhancement imaging to exclude the possibility of myocardial infarction as the cause of the wall motion abnormalities (this is useful even after angiography as rarely, small infarcts can be 
seen even with an apparently normal coronary angiography).

In the acute setting, CMR can also be used to identify some of the potential complications of takotsubo cardiomyopathy such as thrombus formation or systolic anterior motion of the mitral valve. Finally, CMR can be used to confirm the recovery of left ventricular function and, in selected cases, may be used as a tool in guiding the duration of heart failure therapies (i.e. ACE inhibitors, beta blockers) which are sometimes empirically used for treatment.

One increasing used CMR technique is T2 weighted imaging (T2W) whereby spins with long T2 relaxation time-a property which is directly affected by the tissue biochemistry and water content-will have a brighter signal. This unique ability of CMR to detect areas of myocardial edema has been shown to be valuable in several scenarios. In the setting of acute myocardial infarction, this technique can be used to improve the detection of patients with acute coronary syndrome, to differentiate acute versus chronic MI, and to determine the area of myocardial salvage $[6,7]$. T2W imaging is also an integral component when CMR is used for the evaluation of myocarditis [8]. A natural questions that follows is what are the findings and role of $\mathrm{T} 2 \mathrm{~W}$ CMR in the evaluation of takotsubo cardiomyopathy? Preliminary reports indicate that among patient with takotsubo cardiomyopathy, increased T2 signal can be seen in areas of hypokinesis [9].

In this issue of the International Journal of Cardiovascular Imaging, Joshi et al. provide further evidence to support these findings. In one of the first studies utilizing T2W CMR for the diagnosis and follow-up of patients with takotsubo cardiomyopathy, they studied 8 female patients with this condition (all had negative invasive angiograms and absence of late enhancement imaging). In addition to standard cine imaging and late enhancement imaging, they performed $\mathrm{T} 2$ weighted double inversion recovery fast spin echo (T2W DIR FSE) imaging in order to evaluate for myocardial edema. Furthermore, followup CMR studies were performed for 5 out of the 8 patients (media follow-up of 18 days) in order to evaluate for subsequent changes in $\mathrm{T} 2$ signal.

Consistent with the prior reported experience by Abdel-Aty et al. [9]. Joshi and colleagues found that segments which had akinesis or severe hypokinesis ( $<25 \%$ systolic wall thickening), had higher signal on
T2W imaging suggesting increased edema. [ $85 \%$ of the myocardium in these areas had a signal intensity which was two standard deviations above the mean of the remote normal myocardium versus $35 \%$ in segments with systolic wall thickening $>25 \%$.] They also showed that the T2 signal was highest in myocardial segments with the most impaired function. An important aspect of their study is that all 5 patients which had follow-up CMR scans had normal ejection fraction on follow-up and were found to have a significant reduction in T2 signal (85.9-35\%, $P<.001)$.

There are several limitations which are important to acknowledge when using CMR to assess for the presence of myocardial edema by $\mathrm{T} 2 \mathrm{~W}$ imaging. First, rather than directly measuring $\mathrm{T} 2$, current techniques rely on comparing the signal intensity to other areas of normal muscle which do not have edema. In part this is because even for a given patient, there are many other parameters which influence signal intensity and thus a consistent absolute threshold value cannot be utilized. Second, artifacts related to motion, volume averaging, and inability to null areas of static blood can result in areas of increased T2W signal [7, 10]. Realizing these limitations, Joshi and colleagues took special care to measure the signal intensity in the epicardial half of the myocardium (i.e. away from areas of stasis). However, even using such meticulous techniques, signal averaging which is inherent when displaying a single still image which is obtained during myocardial motion cannot be completely avoided.

Despite these technical limitations, this study does convincingly shows that among patients with takotsubo cardiomyopathy, myocardial edema is present in dysfunctional myocardial segments. However, the reason for and implications for these findings is less clear. Edema is a non-specific finding which can be present in the setting of ischemia, myocarditis, infiltrative heart disease and other forms of myocardial injury. Given the aforementioned pathogenesis takotsubo cardiomyopathy, it is possible that the observed increased edema is a direct result of the toxic effects of increased catecholamine levels but other possibilities such as ischemia due to microvascular impairement is also possible.

Clearly, future studies assessing the pathophysiology of takotsubo cardiomyopathy are needed and such investigations, in addition to advancing our understanding regarding the cause and treatment of 
this disorder, may allow us to better understand the reason for the increased $\mathrm{T} 2 \mathrm{~W}$ signal. For the time being, CMR will remain a powerful tool for the evaluation of patients with suspected takotsubo cardiomyopathy, especially when other alternative pathologic processes need to be more definitely excluded. In light of this, it is essential for clinicians to know the CMR features of this condition. The study by Joshi et al expands our knowledge by including the presence of edema as a transient imaging feature found in takotsubo cardiomyopathy.

In conclusion, CMR is uniquely suited for the evaluation of patients with suspected takotsubo cardiomyopathy. In addition to identifying the typical regional wall motion abnormalities involving the apex and/or mid ventricle, it allows a precise quantification of right and left ventricular function, enables the assessment of myocardial perfusion and can be used to exclude other disease processes that mimic this condition. At the same time, and as has been shown for other acute disease processes, CMR can now be used to demonstrate the presence of edema in the setting of takotsubo cardiomyopathy-a finding that possibly will help us better understand, or at least further investigate, the pathophysiology of this rare and fascinating condition.

\section{References}

1. Wittstein IS et al (2005) Neurohumoral features of myocardial stunning due to sudden emotional stress. N Engl J Med 352(6):539-548
2. Ueyama T et al (2002) Emotional stress induces transient left ventricular hypocontraction in the rat via activation of cardiac adrenoceptors: a possible animal model of 'takotsubo' cardiomyopathy. Circ J 66(7):712-713

3. Akashi YJ et al (2008) Takotsubo cardiomyopathy: a new form of acute, reversible heart failure. Circulation 118(25):2754-2762

4. Lyon AR et al (2008) Stress (Takotsubo) cardiomyopathy - a novel pathophysiological hypothesis to explain catecholamine-induced acute myocardial stunning. Nat Clin Pract Cardiovasc Med 5(1):22-29

5. Prasad A, Lerman A, Rihal CS (2008) Apical ballooning syndrome (Tako-Tsubo or stress cardiomyopathy): a mimic of acute myocardial infarction. Am Heart J 155(3):408-417

6. Cury RC et al (2008) Cardiac magnetic resonance with T2weighted imaging improves detection of patients with acute coronary syndrome in the emergency department. Circulation 118(8):837-844

7. Pennell D (2006) Myocardial salvage: retrospection, resolution, and radio waves. Circulation 113(15):1821-1823

8. Friedrich MG et al (2009) Cardiovascular magnetic resonance in myocarditis: a JACC White Paper. J Am Coll Cardiol 53(17):1475-1487

9. Abdel-Aty H, Cocker M, Friedrich MG (2009) Myocardial edema is a feature of Tako-Tsubo cardiomyopathy and is related to the severity of systolic dysfunction: insights from T2-weighted cardiovascular magnetic resonance. Int $\mathrm{J}$ Cardiol 132(2):291-293

10. Kellman P et al (2007) T2-prepared SSFP improves diagnostic confidence in edema imaging in acute myocardial infarction compared to turbo spin echo. Magn Reson Med 57(5):891-897 